\title{
Corrigendum to "Influence of a 10-Day Mimic of Our Ancient Lifestyle on Anthropometrics and Parameters of Metabolism and Inflammation: The "Study of Origin"”
}

\author{
Leo Pruimboom, ${ }^{1,2}$ Begoña Ruiz-Núñez, ${ }^{2}$ Charles L. Raison, ${ }^{3}$ \\ Frits A. J. Muskiet, ${ }^{2}$ and Jens Freese ${ }^{4}$ \\ ${ }^{1}$ Natura Foundation, 3281 NC Numansdorp, Netherlands \\ ${ }^{2}$ Laboratory Medicine, University Medical Center Groningen (UMCG) and University of Groningen, 9713 GZ Groningen, Netherlands \\ ${ }^{3}$ Department of Psychiatry, College of Medicine, John and Doris Norton School of Family and Consumer Sciences, \\ Tucson, AZ 85719, USA \\ ${ }^{4}$ German Sports University Cologne, Cologne, Germany
}

Correspondence should be addressed to Leo Pruimboom; cpni.pruimboom@icloud.com

Received 3 November 2016; Accepted 7 November 2016; Published 23 March 2017

Copyright (C) 2017 Leo Pruimboom et al. This is an open access article distributed under the Creative Commons Attribution License, which permits unrestricted use, distribution, and reproduction in any medium, provided the original work is properly cited.

In the article titled "Influence of a 10-Day Mimic of Our Ancient Lifestyle on Anthropometrics and Parameters of Metabolism and Inflammation: The "Study of Origin"” [1], Jens Freese was missing from the authors' list. The corrected authors' list is shown above.

Also, there was a typographical error in the value of body weight loss. As a result, in the second paragraph of
"Anthropometrics and Clinical Chemical Indices" section, the sentence "We found (Table 1) that body weight decreased with a median (range) of $-3.8 \mathrm{~kg}(-12.5$ to -0.7$)$ " should be changed to "We found (Table 1) that body weight decreased with a median (range) of $-3.4 \mathrm{~kg}(-7.5$ to -0.7$)$," and Table 1 should be corrected as follows:

TABLE 1: Anthropometrics and clinical chemical indices at baseline and at the study end.

\begin{tabular}{|c|c|c|c|c|c|c|c|c|c|c|c|}
\hline & \multirow[b]{2}{*}{ Unit } & \multirow[b]{2}{*}{$N$} & \multicolumn{2}{|c|}{ Baseline } & \multicolumn{2}{|c|}{ Study end } & \multicolumn{4}{|c|}{ Change } & \multirow[b]{2}{*}{$p$ value } \\
\hline & & & Median & Range & Median & Range & Median & Range & $\mathrm{SD}$ & $\begin{array}{l}95 \% \text { CI of the } \\
\text { mean }\end{array}$ & \\
\hline Body weight & $\mathrm{kg}$ & 55 & 68.0 & $48.4-116.3$ & 65.00 & $46.9-111.8$ & -3.4 & -7.5 to -0.7 & 2.0 & -4.4 to -3.3 & $<0.001^{*}$ \\
\hline Age & Years & 50 & 38 & $22-67$ & NM & NM & NM & NM & NM & NM & NM \\
\hline Height & $\mathrm{cm}$ & 55 & 175 & 154-203 & NM & NM & NM & NM & NM & NM & NM \\
\hline BMI & $\mathrm{kg} / \mathrm{m}^{2}$ & 55 & 22.40 & $17.4-31.9$ & 21.3 & $16.8-30.4$ & -1.2 & -4.4 to -0.2 & 0.6 & -1.4 to -1.1 & $<0.001^{*}$ \\
\hline $\begin{array}{l}\text { Hip } \\
\text { circumference }\end{array}$ & $\mathrm{cm}$ & 44 & 100 & $85-120$ & 96 & $86-115$ & -3 & -17 to 5 & 3.3 & -4.2 to -2.2 & $<0.001^{*}$ \\
\hline $\begin{array}{l}\text { Waist } \\
\text { circumference }\end{array}$ & $\mathrm{cm}$ & 44 & 81 & $66-110$ & 76 & 63-101 & -5 & -18 to 9 & 5.5 & -7 to -4 & $<0.001^{*}$ \\
\hline Waist/hip ratio & $\mathrm{cm} / \mathrm{cm}$ & 44 & 0.84 & $0.72-1.00$ & 0.80 & $0.66-0.94$ & -0.02 & -0.14 to 0.10 & 0.06 & $\begin{array}{l}-0.04 \text { to } \\
-0.02\end{array}$ & $<0.002^{*}$ \\
\hline
\end{tabular}


TABLE 1: Continued.

\begin{tabular}{|c|c|c|c|c|c|c|c|c|c|c|c|}
\hline & \multirow[b]{2}{*}{ Unit } & \multirow[b]{2}{*}{$N$} & \multicolumn{2}{|c|}{ Baseline } & \multicolumn{2}{|c|}{ Study end } & \multicolumn{4}{|c|}{ Change } & \multirow[b]{2}{*}{$p$ value } \\
\hline & & & Median & Range & Median & Range & Median & Range & SD & $\begin{array}{c}95 \% \text { CI of the } \\
\text { mean }\end{array}$ & \\
\hline Glucose & $\mathrm{mmol} / \mathrm{L}$ & 53 & 4.9 & $4.2-5.8$ & 4.3 & $3.3-6.1$ & -0.6 & -1.7 to 0.5 & 0.6 & -0.8 to -0.5 & $<0.001^{*}$ \\
\hline HbAlc & $\%$ & 53 & 5.3 & $4.8-6.1$ & 5.3 & $4.7-6.1$ & -0.1 & -0.4 to 0.2 & 0.2 & -0.1 to -0.05 & $<0.001^{*}$ \\
\hline Insulin & $\mathrm{mU} / \mathrm{L}$ & 23 & 14.0 & $3.7-36.8$ & 6.7 & $1.1-12.9$ & -4.7 & -31.4 to -0.2 & 8.1 & -12.2 to -5.2 & $<0.001^{*}$ \\
\hline HOMA-IR & $\mathrm{mmol} * \mathrm{mU} / \mathrm{L}^{2}$ & 22 & 3.0 & $0.8-7.9$ & 1.4 & $0.2-2.6$ & -1.2 & -7.0 to -0.4 & 1.8 & -2.8 to -1.3 & $<0.001^{*}$ \\
\hline Triglycerides & $\mathrm{mmol} / \mathrm{L}$ & 53 & 0.69 & $0.34-6.68$ & 0.52 & $0.37-2.77$ & -0.14 & -6.12 to 2.18 & 0.92 & -0.52 to -0.01 & $<0.001^{*}$ \\
\hline $\begin{array}{l}\text { Total } \\
\text { cholesterol }\end{array}$ & $\mathrm{mmol} / \mathrm{L}$ & 53 & 5.2 & $3.2-8.2$ & 4.5 & $2.6-8.1$ & -0.7 & -2.8 to 0.4 & 0.7 & -1.0 to -0.6 & $<0.001^{*}$ \\
\hline $\begin{array}{l}\text { HDL- } \\
\text { cholesterol }\end{array}$ & $\mathrm{mmol} / \mathrm{L}$ & 53 & 2.0 & $0.7-3.1$ & 1.9 & $1.0-3.5$ & 0.0 & -0.8 to 0.6 & 0.3 & -0.1 to 0.1 & 0.464 \\
\hline $\begin{array}{l}\text { LDL- } \\
\text { cholesterol }\end{array}$ & $\mathrm{mmol} / \mathrm{L}$ & 52 & 3.0 & $1.3-5.8$ & 2.5 & $0.0-5.4$ & -0.6 & -3.1 to 0.6 & 0.7 & -0.8 to -0.5 & $<0.001^{*}$ \\
\hline $\begin{array}{l}\text { TG/HDL- } \\
\text { cholesterol } \\
\text { ratio }\end{array}$ & $\mathrm{mol} / \mathrm{mol}$ & 53 & 0.3 & $0.16-9.54$ & 0.26 & $0.11-1.73$ & -0.55 & -8.98 to 1.34 & 1.3 & -0.59 to 0.98 & $<0.001^{*}$ \\
\hline ASAT & $\mathrm{IU} / \mathrm{L}$ & 53 & 22 & $14-52$ & 33 & $11-75$ & 11 & -8 to 54 & 11.4 & 9 to 15 & $<0.001^{*}$ \\
\hline ALAT & $\mathrm{IU} / \mathrm{L}$ & 53 & 20 & $11-42$ & 25 & $12-47$ & 6.0 & -13 to 52 & 7.3 & 5 to 9 & $<0.001^{*}$ \\
\hline CRP & $\mathrm{mg} / \mathrm{L}$ & 42 & 0.61 & $0.14-27.04$ & 1.36 & $0.14-41.65$ & 0.56 & -15.72 to 41.07 & 8.45 & 0.20 to 5.46 & $<0.001^{*}$ \\
\hline $\mathrm{TSH}$ & $\mathrm{mU} / \mathrm{L}$ & 42 & 1.25 & $0.02-3.12$ & 1.11 & $0.02-4.40$ & -0.08 & -0.93 to 1.28 & 0.47 & -0.19 to -0.10 & 0.326 \\
\hline FT4 & $\mathrm{pmol} / \mathrm{L}$ & 42 & 10.8 & $7.9-19.4$ & 11.3 & $7.8-20.6$ & 0.1 & -5.6 to 8.4 & 2.3 & -0.4 to 1.1 & 0.378 \\
\hline FT3 & $\mathrm{pmol} / \mathrm{L}$ & 42 & 4.4 & $2.3-6.5$ & 3.5 & $1.7-8.7$ & -0.8 & -3.4 to 3.1 & 1.0 & -1.0 to -0.5 & $<0.001^{*}$ \\
\hline
\end{tabular}

Data are medians (range). ALAT, alanine aminotransferase; ASAT, aspartate aminotransferase; BMI, body mass index; CRP, C-reactive protein; FT3, free triiodothyronine; FT4, free thyroxine; HbAlc, hemoglobin Alc; HDL, high-density lipoprotein; HOMA-IR, homeostasis model assessment-estimated insulin resistance; LDL, low-density lipoprotein; NM, not measured; TG, triglycerides; TSH, thyroid-stimulating hormone. * Significant difference between the values before and after the intervention by Wilcoxon signed rank test at $p<0.05$.

\section{References}

[1] L. Pruimboom, B. Ruiz-Núñez, C. L. Raison, and F. A. J. Muskiet, "Influence of a 10-day mimic of our ancient lifestyle on anthropometrics and parameters of metabolism and inflammation: the "study of origin"," BioMed Research International, vol. 2016, Article ID 6935123, 9 pages, 2016. 

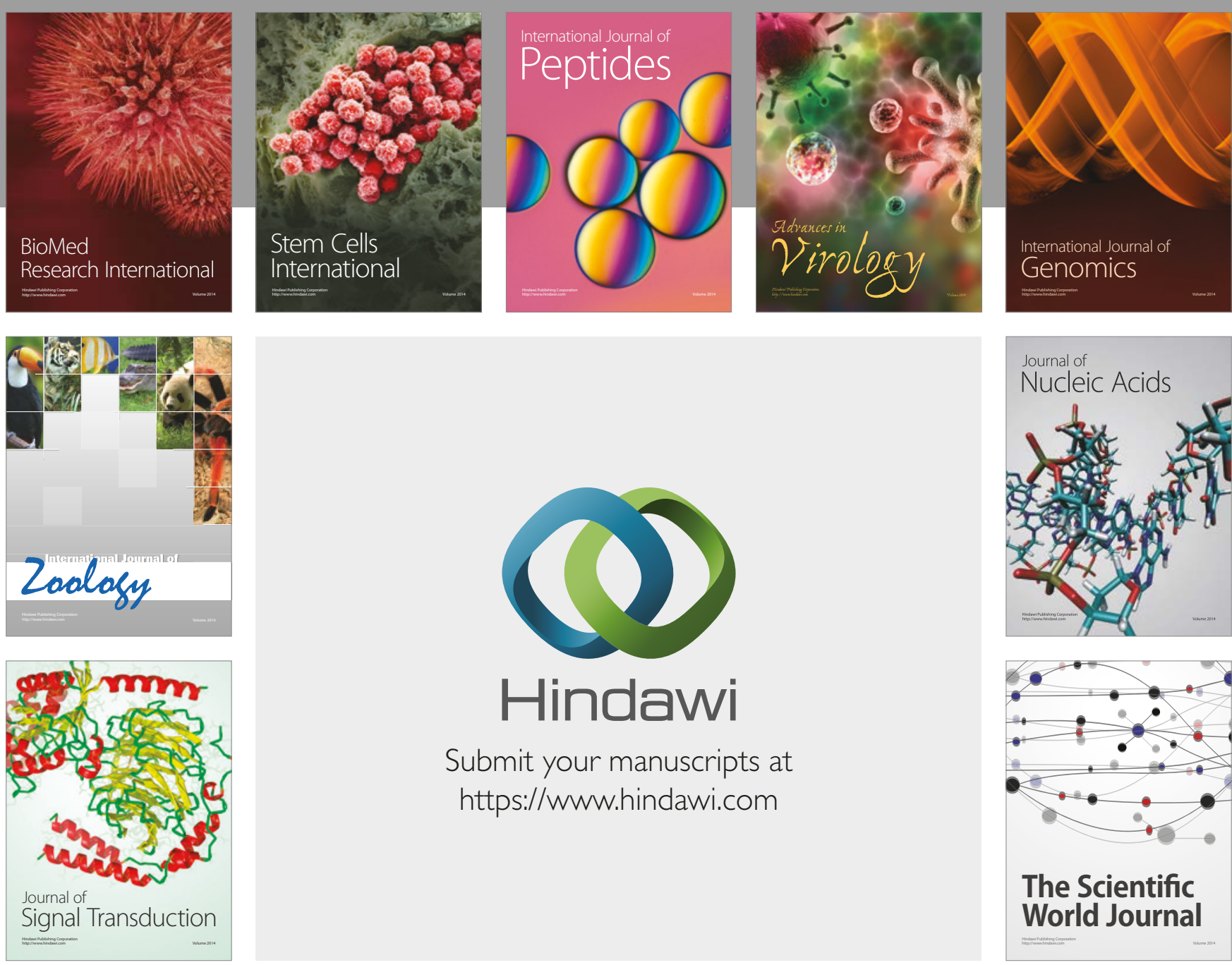

Submit your manuscripts at

https://www.hindawi.com
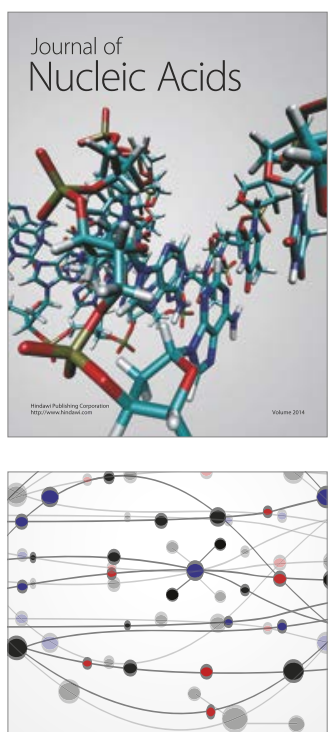

The Scientific World Journal

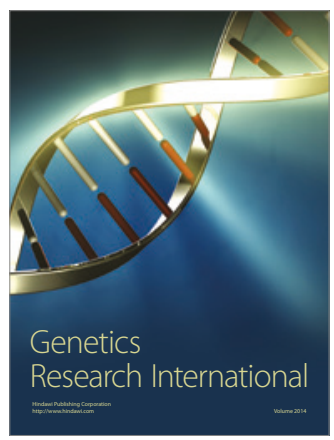

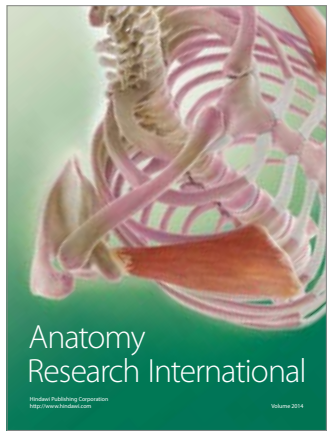

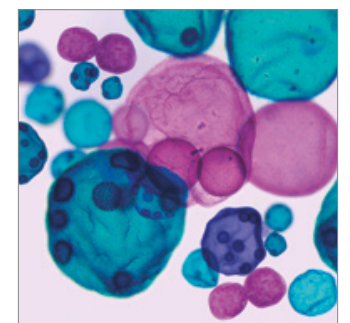

International Journal of Microbiology
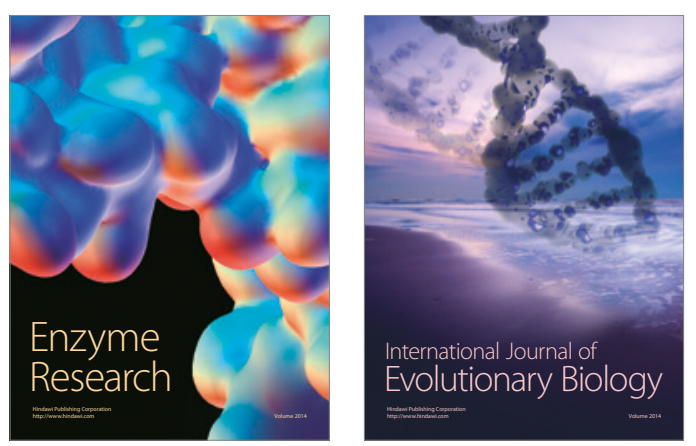
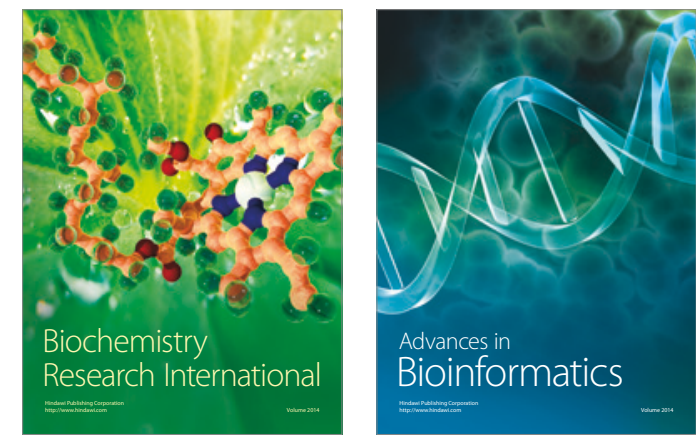

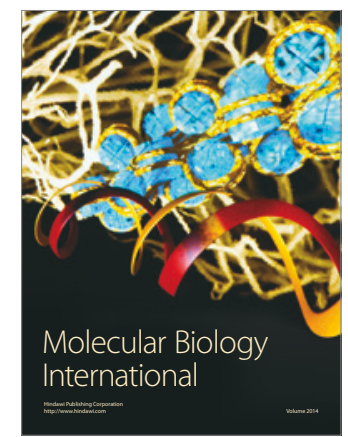

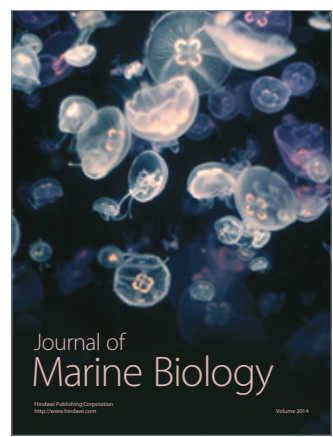

